\title{
Propagation of lead in the human body
}

\author{
Melissa Morrissey * \\ Division of Applied Mathematics \\ Brown University \\ Providence, RI 02912
}

\author{
Jordan Collignon ${ }^{\dagger}$ \\ Department of Mathematics and Statistics \\ California State University, Monterey Bay \\ Marina, CA 93933
}

\author{
Faculty Advisor: Todd Kapitula $\ddagger$ \\ Department of Mathematics and Statistics \\ Calvin College \\ Grand Rapids, MI 49546 \\ Graduate Assistant: Veronica Ciocanel ${ }^{\S}$ \\ Division of Applied Mathematics and ICERM \\ Brown University \\ Providence, RI 02912
}

June 29, 2017

\begin{abstract}
Lead is a toxin that has well known side effects including fatigue, muscle pain, impaired kidney function, lower IQs for children, and brittle bones. Lead can be absorbed into the body through paint, air, water, and various other consumer products. Once ingested, blood transports lead throughout the body. The vast majority of lead absorbed in the body accumulates in the bone. Here we explore a three-compartment nonlinear ODE model for lead in blood, cortical bone, and trabecular bone. Thereafter, we compare the ODE results with a PDE model in which it is assumed that lead slowly diffuses through the bone. Numerical solutions of the model ODE equations suggest that in order to have results which are consistent with experimental data, one should assume nonlinear interactions between the blood and cortical bone, but linear interactions between the blood and trabecular bone. On the other hand, we find that the PDE model we use does not provide for a good comparison with the data. We briefly touch upon some possible reasons for this discrepancy, and ways in which the model could be improved.
\end{abstract}

Keywords. Lead dynamics, nonlinear ordinary differential equations, nonlinear partial differential equations

\section{Contents}

2. The transfer rates 84

3. The ODE model 85

3.1. The steady state solutions . . . . . . . . . . . . . . . . . . . . . 86

3.2. Comparison of linear model with nonlinear model . . . . . . . . . . . . . . . . . . . . . . 88

\footnotetext{
*E-mail: melissa_morrissey@brown.edu

${ }^{\dagger}$ E-mail: jcollignon@csumb.edu

${ }^{\ddagger}$ E-mail: tmk5@calvin.edu

${ }^{\S}$ E-mail: veronica_ciocanel@alumni.brown.edu
} 
4. The PDE model 91

4.1. Simulation results . . . . . . . . . . . . . . . . . . . . . . . . . . 994

4.2. Comparison to ODE model . . . . . . . . . . . . . . . . . . . . . 95

5. Conclusions and Further Work 96

References 


\section{INTRODUCTION}

Lead is ingested into the body in various ways including contaminated air, water, food, soil, and other consumer products [19]. After ingestion, blood takes the lead into the body and then transports it to other tissues and the bones [11]. More often, lead is released from the blood under periods of change such as during pregnancy, aging, and osteoporosis. The lead accumulates and stays in the bone for decades [23]. It is also evident that lead affects different populations differently, such as children versus adults and males versus females $[\mathbf{1}]$.

High amounts of lead released back into the blood can cause lead poisoning which can increase the risk of symptoms such as fatigue, muscle pain, impaired kidney function, reduced nerve conduction velocity and inhibited central nervous system [9]. Lead absorption in the skeletal system can also impede bone growth and make the bones brittle. With age, bones lose calcium and other minerals, and individuals may develop thinner cortical bone and fewer and thinner trabeculae, while at the same time bone density and elasticity decreases [6]. Additionally mineral density, bone volume fraction and trabecular number decreases. This process happens quicker for women [6]. There can also be damage to the brain for both children and adults while interfering with the production of red blood cells that carry oxygen throughout the body. Individuals at greatest risk include infants, young children and pregnant women. Adults at higher risk also include those with poor kidney function and high blood pressure [21]. Previous research and experiments indicate that any amount of ingested lead in the human body can be dangerous [11].

In this work, our goal is to provide insight into the accumulation of lead in the body with time, given a constant rate of ingestion. While a constant rate of ingestion is likely not physically possible, this assumption simplifies our model analysis. To achieve this, we explore a three-compartment model for lead in blood, cortical bone and trabecular bone (see Figure 1, which is a cartoon of $[\mathbf{4}$, Fig. 1]). Experimentally it is known that at least $90 \%$ of lead is stored in the bone rather than in other tissue [2]. This is because the majority of lead settles where calcium deposits are found [17]. Similar to established models such as [5], here we separate the bones into two separate compartments because the transfer rates between blood and the two bone types are reported to be significantly different [4]. As Figure 2 illustrates, the tougher cortical bone has a lower transfer rate to the blood than the spongier trabecular bone when the amount of lead in the bone is less than $50 \mathrm{mg}$; otherwise, the rates are comparable.

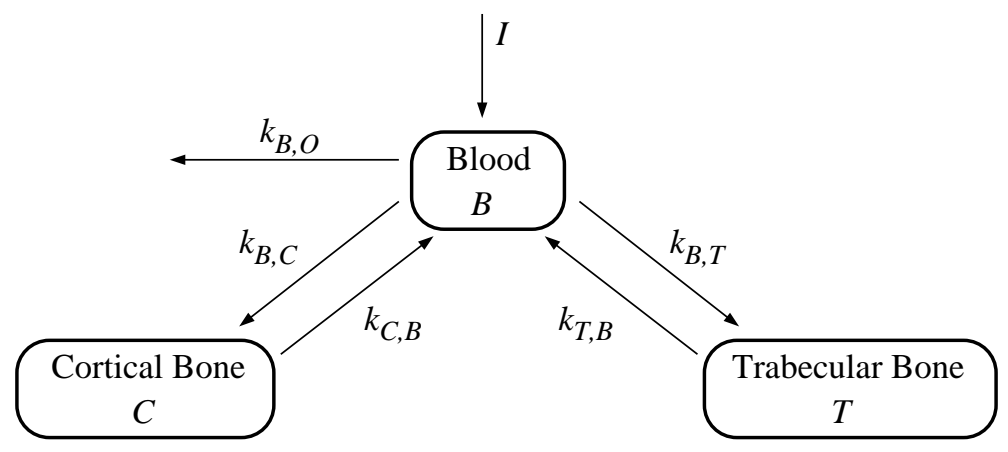

Figure 1: The three compartment model considered in this text. The value of $I$ represents the lead ingestion rate, and the experimental rate values $k_{\star, \star}$ are determined using data from [4] $(\star=$ B: blood, C: cortical bone, or T: trabecular bone). Tissues and other body compartments are not included in this model. Rates are measured in units of 1 /day, and B, C, T are measured in mg.

Our goal is to determine if nonlinearity and/or diffusion (as suggested by Marcus [14]) play an important role in a dynamical compartment model of lead propagation. In most studies, the transfer rates between compartments are assumed to be constant; however, these rates may in fact be nonlinear given the dependence upon the amount of lead in the system (it is often assumed that the threshold for nonlinearity is a concentration of $0.25 \mathrm{mg} / \mathrm{lit}$ of lead in the blood). Diffusion may play a role as it models the process of lead migration through the bone via calcium replacement. 
Previous studies consider different numbers of compartments and various corresponding ODE models. The EPA technical report [22] (also see the references in Section 4.0) provides for an excellent overview of several models which are currently being used. The Rabinowitz model is a linear compartment ODE which divides the body into three compartments: bone, blood, and soft tissue. For the given rate constants it predicts that $98 \%$ of the lead in the body will be found in the bone. The Bert model is a more complicated four compartment linear ODE model which breaks the bone compartment of the Rabinowitz model into two compartments: cortical bone, and trabecular bone. In this model the two bone compartments are distinguished by the transfer rate between blood and bone; in particular, the rate is larger for trabecular bone than it is for cortical bone. The Leggett model is a compartment model which breaks the two bone compartments into four compartments; in particular, each bone type has a surface compartment, and a volume compartment. The idea is that the exchange rates will be different for the subcompartments; in particular, the rate for the surface will be larger than the rate for the volume. An interesting additional feature to this model is that the rate constants change as a function of time. Finally, nonlinearity was introduced through the mechanism of lead uptake by red blood cells. This nonlinearity does not seem to have any effect on lead concentration in the bones, however. Finally, the O'Flaherty model is an extension of the previous models which simulates the delivery of lead to the tissues as a function of blood flow to the tissues. It is similar to the Rabinowitz model in that it treats the bone as one compartment; on the other hand, instead of there being one compartment for the tissue, there are five. An underlying model assumption is that all lead in plasma is available to exchange with tissues. O'Flaherty eventually revised the model to include effects such as different bone types, differences between lead exchange on the bone surface via a leadcalcium exchange having to do with bone formation and resorption vs. an internal plasma-bone interaction. Moreover, the bone type is allowed to be different (juvenile vs. mature), and the rate constants are again assumed to be a function of time. It does not appear that the model allows for nonlinear interactions. Finally, the models of $[\mathbf{5}, \mathbf{1 6}]$ mention that nonlinearity comes into play when people consume high amounts of lead, but unfortunately that threshold is not specified.

The paper is organized as follows. In Section 2 we derive the transfer rates between bone and blood based on data available in the literature. In Section 3 we describe the three compartment ODE model based off Figure 1, and compare the predictions associated with the linear model with those coming from the nonlinear model. In Section 4 we introduce spatial effects by looking at a bone fragment which is assumed to be a cylinder, and assuming that the dynamics occur only in the radial direction. A key feature of the model is that we must take into account the fact that the blood interacts with the bone only in a small inner radius, while diffusion has an effect on the dynamics outside this inner cylinder. Finally, we conclude with some potential directions for future work.

\section{THE TRANSFER RATES}

We define the variables $\mathrm{C}, \mathrm{T}$, and $\mathrm{B}$ to represent the amount of lead as a function of time in the cortical bone, trabecular bone, and blood, respectively, measured in mg. Recalling the cartoon of Figure 1 describing the model compartments, we have:

(a) $k_{B, O}$ is the rate at which lead leaves the body

(b) $k_{B, C}$ is the transfer rate from the blood to the cortical bone

(c) $k_{B, T}$ is the transfer rate from the blood to the trabecular bone

(d) $k_{C, B}$ is the transfer of lead from the cortical bone to the blood

(e) $k_{T, B}$ is the transfer of lead from the trabecular bone to blood.

The transfer rates from the blood to the various bone compartments are fixed and independent of the lead concentration. However, data in [4] shows that the transfer rates from the bone back to the blood do depend upon the levels of lead (see Figure 2). These rates are estimated from measurements of lead content in the various compartments. Note that the transfer rates are decreasing nonlinearly as the amount of lead increases (see [4, Fig. 5 and Fig. 6]). The decrease is due to the fact that at higher bone lead concentrations 
the formation of new bone is delayed due to the presence of lead, which causes the lead to stay longer in the bone. In those figures the transfer rates were estimated after first experimentally determining the lead content in the various compartments. The data was extracted from the article using WebPlotDigitizer, and then imported into a file which could be read into MATLAB.

Marcus [15] suggests a rate for site-limited blood uptake of the form,

$$
k_{C, B} \mapsto\left\{\begin{array}{l}
k_{C, B} /\left(1+P_{C, B} C\right), \\
k_{C, B}\left(1-P_{C, B} C\right) .
\end{array}\right.
$$

In [15], the meaning of $P_{C, B}$ is not given, however the first expression is justified as a rate-limited blood uptake, while the second is introduced as a site-limited blood uptake. We note that the second expression for $k_{C, B}$ is simply the linear approximation to the first expression. An examination of the log-log plot of the data plotted in Figure 2 suggests that we fit the log-log data with a line. We chose the following functional form for the transfer rates:

$$
\ln \left(k_{\star, B}\right) \mapsto \ln \left(k_{\star, B}\right)-P_{\star, B} \ln (1+\star), \quad \star \in\{C, T\},
$$

which provides for a power law for the rates,

$$
k_{\star, B} \mapsto \frac{k_{\star, B}}{(1+\star)^{P_{\star}, B}} .
$$

Similarly, $[\mathbf{1 5}]$ proposes a model of increased plasma clearance where the transfer rate from the blood to outside the body increases as a function of the level of lead in the blood: $k_{B, O} \mapsto k_{B, O}\left(1+P_{B, O} B\right)$. We assume that the transfer rate for large amounts of lead in the blood has an upper bound of twice the rate for small amounts, which provides for a model of the form,

$$
k_{B, O} \mapsto k_{B, O}\left[1+\tanh \left(P_{B, O} B\right)\right] .
$$

Fitting the data in Figure 2 to the nonlinear expressions in equation (2.1) yields the parameters reported in the table below. Since

$$
k_{C, B} \sim 2.894 \times 10^{-4}, \quad k_{T, B} \sim 7.312 \times 10^{-4},
$$

our estimating curve recovers the expected result that the linear exchange rate from the softer bone to the blood is larger than the rate from the harder bone to the blood. However, the exchange rates become comparable once each bone segment contains roughly $50 \mathrm{mg}$ of lead. For the values of $k_{B, T}$ and $k_{B, C}$ we used the average in the range of values provided in [4]. Given the absence of data for transfer of lead from blood to outside the body, we arbitrarily chose $P_{B, O}=0.005$ in our simulations. Of course, other choices would affect the following quantitative results, but the qualitative results will remain essentially the same.

Remark 2.1. The model is linear if $P_{C, B}=P_{T, B}=P_{B, O}=0$. It is generally assumed that if the concentration of lead in the blood stays below $0.25 \mathrm{mg} /$ lit (which yields a total of $1.35 \mathrm{mg}$ of lead in the blood for an adult who has approximately 5.4 liters of blood in the body), then the dynamics are essentially governed by the linear model.

\begin{tabular}{|c|c|c||c|c||c|c|}
\hline$k_{B, O}$ & $k_{B, C}$ & $k_{B, T}$ & $k_{C, B}$ & $P_{C, B}$ & $k_{T, B}$ & $P_{T, B}$ \\
\hline 0.01555 & 0.0023 & 0.0019 & $\exp (-8.1478)$ & 0.2462 & $\exp (-7.2208)$ & 0.4618 \\
\hline
\end{tabular}

Table 1: The constants associated with the models for the interaction rate functions. The constants $k_{\star, B}$ for $\star \in\{C, T\}$ are the linear rates for transfer from bone to blood and are measured in $1 /$ day, while the constants $P_{\star, B}$ are the exponents for the assumed power law (see (2.2)).

\section{The ODE MODEL}

Assuming the amount of lead in each compartment is uniformly distributed (i.e., the concentration within a particular compartment is spatially independent), the compartmental model leads to the following system 

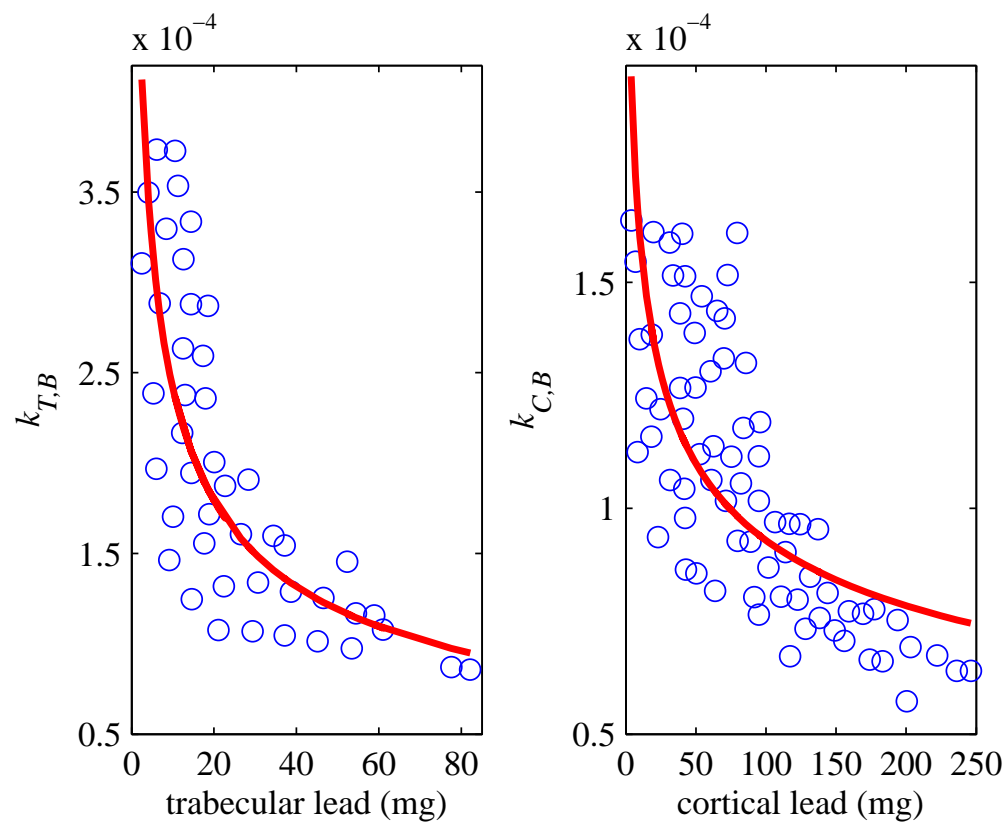

Figure 2: The exchange rates (see [4]) for lead exchange from bone (trabecular in the left panel, and cortical in the right panel) as a function of amount of lead (mg) in the bone are shown by circles. The time scale is 1 day, so that the transfer rates are measured in units of $1 /$ day. The curve of best fit is given by the solid (red) curve. The logarithm of the exchange rate vs. amount of lead in each in each figure is fit with a curve of the functional form $a+b \ln (1+x)$.

of ordinary differential equations:

$$
\begin{aligned}
& \frac{\mathrm{d} B}{\mathrm{~d} t}=-\left(k_{B, C}+k_{B, T}+k_{B, O}\right) B+k_{C, B} C+k_{T, B} T+I \\
& \frac{\mathrm{d} C}{\mathrm{~d} t}=k_{B, C} B-k_{C, B} C \\
& \frac{\mathrm{d} T}{\mathrm{~d} t}=k_{B, T} B-k_{T, B} T,
\end{aligned}
$$

where $\mathrm{T}, \mathrm{B}, \mathrm{C}$ correspond to the amount of lead as described in $\S 2$, I is the ingestion rate of lead in the units of $\mathrm{mg} /$ day, and the meaning of the rates is given in $\S 2$ (see also [4, Section 2.2]). The equations are linear if the transfer rates are independent of the concentration of lead, and nonlinear otherwise. The linear equations will have $P_{C, B}=P_{T, B}=P_{B, O}=0$. We note that the model proposed is symbolically identical to the one in [4]. However, while [4] uses this approach to carry out optimization and parameter estimation based on experimental data, our goals are to examine the steady states of the system in the case of linear and nonlinear reaction rates as well as the amount of lead variation in each compartment with time.

\subsection{The steady state solutions}

We first examine the steady state solutions to the linear and nonlinear systems in order to determine the effect of nonlinearity. The linear system corresponds to using the values presented in the table for the transfer rate coefficients, while setting $P_{C, B}=P_{T, B}=P_{B, O}=0$. The expectation is that for small levels of lead the 


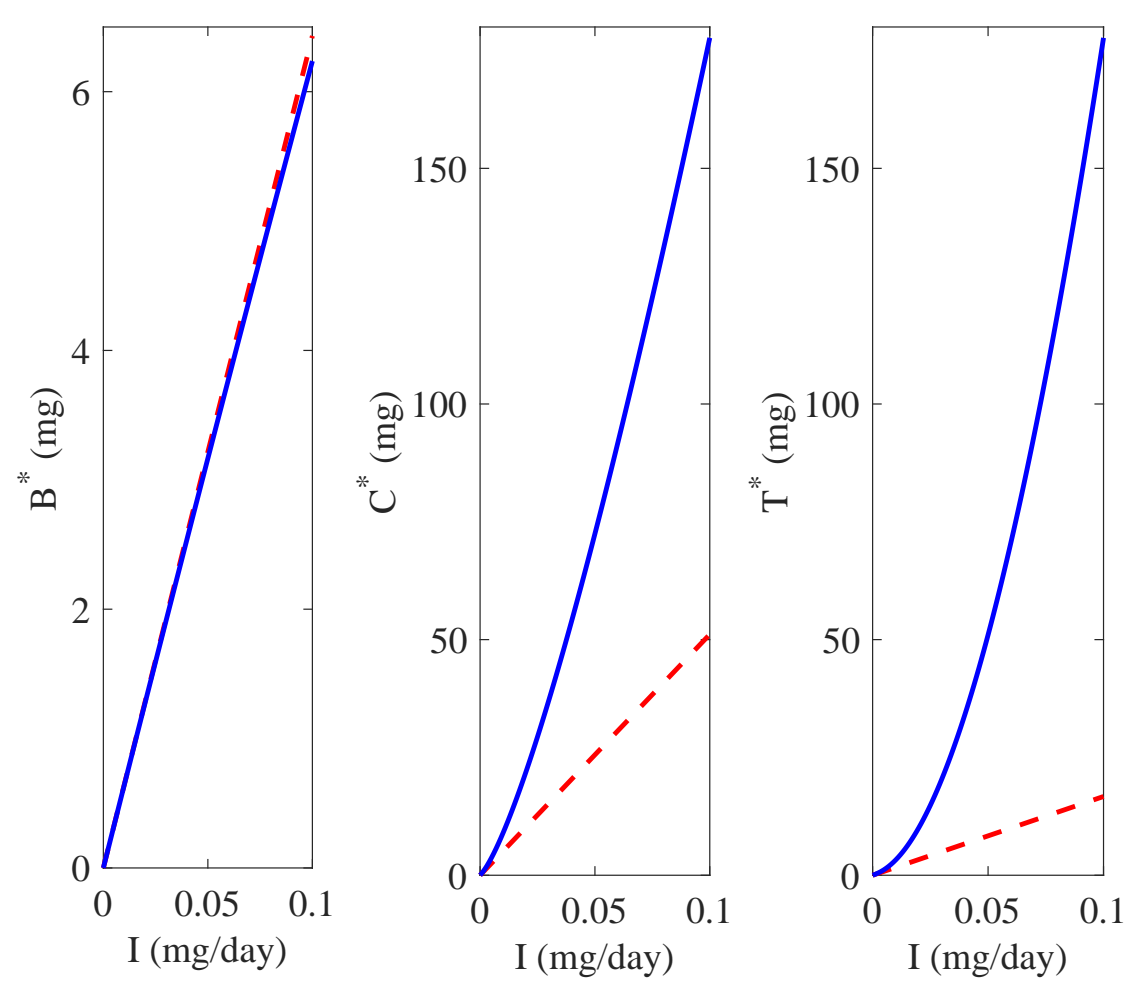

Figure 3: Linear vs. nonlinear steady states. The linear steady states are denoted by a dashed (red) line, and the nonlinear steady states are given by a solid (blue) line.

linear and nonlinear solutions should coincide. The linear case has only one analytical solution,

$$
\begin{aligned}
B^{*} & =\frac{1}{k_{B, O}} I \sim 64.3 I \\
C^{*} & =\frac{k_{B, C}}{k_{B, O} k_{C, B}} I \sim 511.1 I \\
T^{*} & =\frac{k_{B, T}}{k_{B, O} k_{T, B}} I \sim 167.1 I .
\end{aligned}
$$

For a given value of $I$ the fractional amounts of the total amount of lead considered in our model are,

$$
B_{\text {frac }} \sim 0.0866, \quad C_{\text {frac }} \sim 0.6884, \quad T_{\text {frac }} \sim 0.2250 .
$$

Roughly $91.3 \%$ of the overall lead is in the bone, but of the lead in the bone, a little more than three times more is in the cortical (hard) bone than is in the trabecular (soft) bone.

The steady state for the nonlinear problem can only be determined numerically, and we use Matlab's nonlinear system solver fsolve. Figure 3 compares the linear and the nonlinear steady states and illustrates that as the ingestion rate increases:

(a) the amount of lead in the blood is slightly smaller for the nonlinear problem as it is for the linear problem

(b) the amount of lead in each of the bone segments is markedly larger for the nonlinear problem as it is for the linear problem.

Given that the transfer rates from bone to blood decrease as the level of lead increases, these conclusions are not surprising. Moreover, the fact that the nonlinear steady state for the amount of lead in the trabecular 
bone increases more quickly as a function of input rate as does that in the cortical bone is primarily due to the fact that the decay rate of the transfer rate for the former is roughly twice that of the latter.

Remark 3.1. As seen in Figure 2 there is data for $k_{T, B}$ only if the amount of lead in the trabecular bone is no more than $85 \mathrm{mg}$. Thus, while we may with some confidence use the approximating rate curve as long as the amount of lead in this bone is smaller than $85 \mathrm{mg}$, it is uncertain how much the curve should be trusted for larger amounts of lead in that bone. Roughly speaking, this limit for the steady state is reached for $I \sim 0.065 \mathrm{mg} /$ day. However, even for this relatively small input rate there is still a marked difference between the linear and nonlinear steady states for both bone segments.

Remark 3.2. Recall that the dynamics are often assumed to be linear as long as the amount of lead in the blood is less than $1.35 \mathrm{mg}$, which as far as the steady states are concerned corresponds to an input rate of approximately $0.022 \mathrm{mg} /$ day. As we see in these steady state plots, even at this relatively small input rate the nonlinear effect associated with the amount of lead in each of the bone segments is already markedly present.

\subsection{Comparison of linear model with nonlinear model}

For the linear problem the proportions given in (3.2) are independent of the incoming rate of lead. On the other hand, for the nonlinear problem the relative proportions do depend on the incoming rate, as is evidenced by the fact that the steady state for each component is a nonlinear function of $I$. Since it takes several lifetimes for the steady state to be achieved, we now focus on the case of constant ingestion of lead over a 50 year period. The assumption is that the body is free of lead at the beginning, so the initial condition for the ODE is $B(0)=C(0)=T(0)=0$.
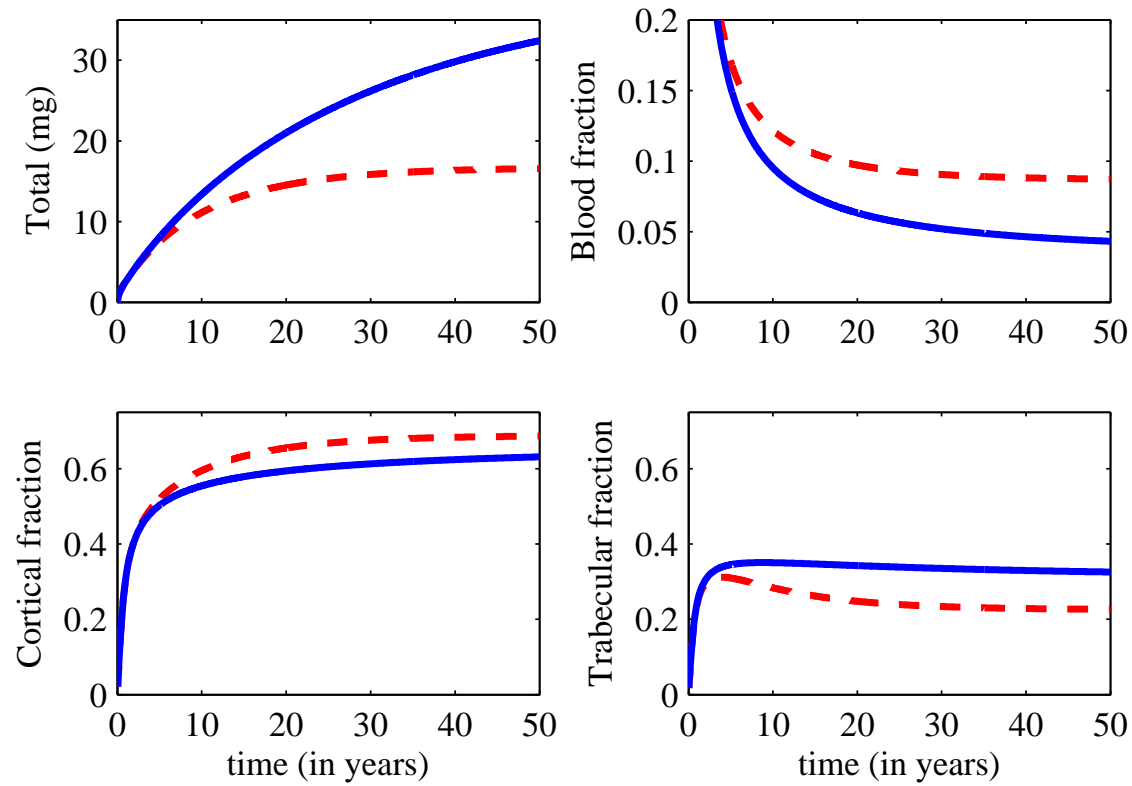

Figure 4: The total amount of lead and the relative fractions of lead in each body compartment when the input rate is $I=0.0225$. The dashed (red) curve corresponds to the linear solution, and the solid (blue) curve corresponds to the nonlinear solution.

For the sake of comparison between the linear and nonlinear models we will consider the ingestion rate of $I=0.0225 \mathrm{mg} /$ day. We choose this rate by assuming the lead is being consumed by drinking 1.5 liters/day of water which contains the federally mandated upper limit of $15 \mathrm{ppb}$ (parts per billion) of lead. The numerical solution to the ODE is given in Figure 4. The dashed (red) curves correspond to solutions for the linear problem, and the solid (blue) curves correspond to solutions for the nonlinear problem. The amount of lead 
in the blood becomes larger than $1.35 \mathrm{mg}$ in less than three years for this input rate.

As anticipated from our steady state analysis, there is a clear difference between the linear and nonlinear model for the total amount of lead in the body once the lead levels get sufficiently large (top left panel). After 50 years the linear model predicts the body will retain $16.6 \mathrm{mg}$, whereas the nonlinear model predicts a retention of $32.4 \mathrm{mg}$. Regarding the relative fractions, even though the total amount of lead in the body continues to increase, after roughly 30 years the relative fractions achieve a steady state. Comparing the linear (L) and nonlinear models (NL), we have after a long time,

(a) blood: $0.0872(\mathrm{~L})$ vs. $0.0431(\mathrm{NL})$

(b) cortical: $0.6864(\mathrm{~L})$ vs. $0.6313(\mathrm{NL})$

(c) trabecular: $0.2264(\mathrm{~L})$ vs. $0.3256(\mathrm{NL})$.

Thus, the nonlinear model not only predicts there will be almost double the amount of lead in the body, but that a larger percentage of it (95.7\% vs. $91.3 \%)$ will reside in the bone. Of that lead in the bone, the nonlinear model predicts that more of it will reside within the trabecular than predicts the linear model. However, as expected due to the smaller exchange rate for lead amounts below $50 \mathrm{mg}$, the majority of the lead is still located in the cortical bone. These numerical results are consistent with the claim that at least $90 \%$ of lead settles in the bone, see $[\mathbf{2}, \mathbf{3}]$.

While we do not show the figures here, our numerical experiments indicate that over this time frame the amount of lead in the blood will become larger than the threshold value of $1.35 \mathrm{mg}$ if $I>0.02$. However, even for smaller values of $I$ we find that,

(a) the nonlinear model predicts the body will have significantly more lead after a lifetime of ingestion

(b) the nonlinear model predicts that a greater percentage of the lead will be in the bone than in the blood

(c) of that lead in the bone, the nonlinear model predicts less of it will be in the hard cortical bone, and more will be in the softer trabecular bone.

Regarding point (b), even for a value as small as $I=0.005$ we find that the relative percentage of lead in the blood is $6.42 \%$, as compared to the linear prediction of $8.72 \%$. The percentages do not coincide until $I \leq 0.001$ (which corresponds to a lead concentration of $6.67 \mathrm{ppb}$ in the water). As for point (c), this is due to the fact that once the amount of lead in the bone segment is above $50 \mathrm{mg}$, then the transfer rates for cortical bone and trabecular bone are comparable.

Remark 3.3. It is interesting to note the difference in the predictions regarding the total amount of lead in the body provided for by the two models. Suppose it is determined that the level of lead in the blood is 200 ppb. Assuming that the body holds 5.4 liters of blood, this means that there are 1.08 milligrams of lead in the blood. Now, using the percentages associated with the time of 30 years, the linear model predicts $9.05 \%$ of the total lead will be in the blood, while the nonlinear model predicts $5.20 \%$ of the total lead will be in the blood. Consequently, the linear model predicts a total of 11.93 milligrams of lead in the body, whereas the nonlinear model predicts a total of 20.77 milligrams of lead in the body.

We now turn our attention to the concentration of lead in each body compartment. Here we assume the volumes and masses associated with an adult human body: 5.4 liters of blood, $2000 \mathrm{~g}$ of cortical bone, and $500 \mathrm{~g}$ of trabecular bone (see [4, Section 2.2]). We still assume the input ingestion rate of $0.0225 \mathrm{mg} /$ day. The results are plotted in Figure 5, where again the dashed (red) curves correspond to the predictions given by the linear ODE model, and the solid (blue) curves correspond to the nonlinear ODE predictions. The linear and nonlinear models essentially agree concerning the concentration of lead in the blood. After a short period of time the level rises to roughly $0.225 \mathrm{ppm}$ for both models, with it being the case that the linear model then predicts there will be a slightly stronger concentration. Both models predict that even after a lifetime of ingestion the concentration will still be well below the level of acute lead poisoning, 0.8-1.0 ppm.

The concentration in each bone compartment is also measured by using the assumed densities of $1.9 \mathrm{~g} / \mathrm{cm}^{3}$ for the harder cortical bone, and $1.2 \mathrm{~g} / \mathrm{cm}^{3}$ for the softer trabecular bone. As we see in the right two panels, the linear and nonlinear models essentially agree over the first five years. This is not surprising, as it takes 

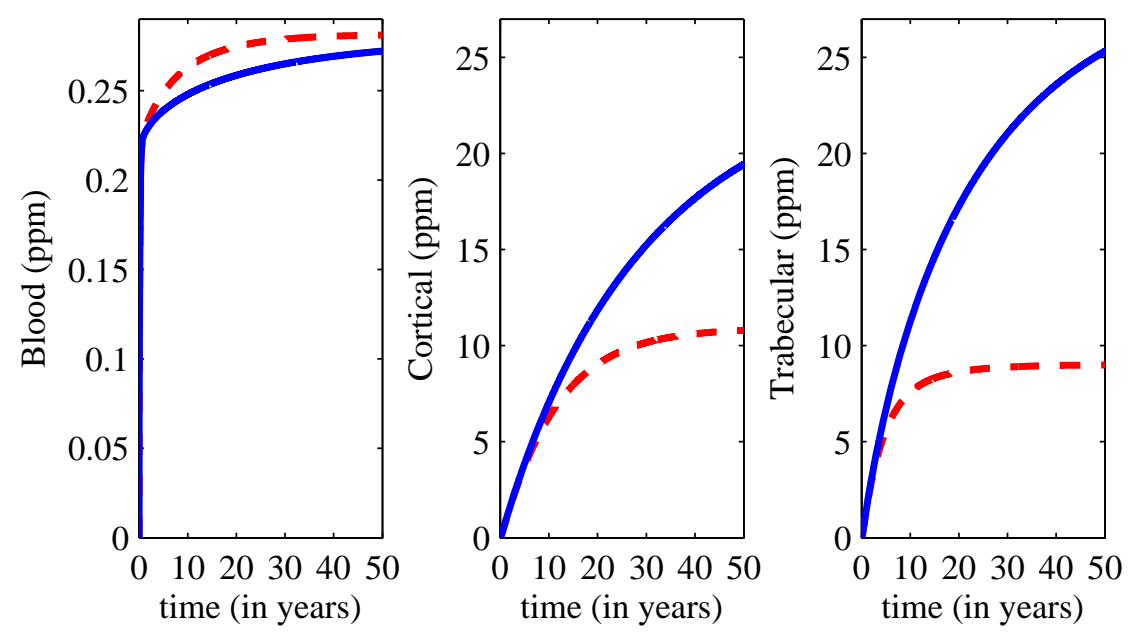

Figure 5: The concentration of lead in each body compartment when the input rate is $I=0.0225$. The dashed (red) curve corresponds to the linear solution, and the solid (blue) curve corresponds to the nonlinear solution.

almost three years before the amount of lead in the blood is large enough for the nonlinear effects of the boneto-blood transfer rates to be felt. After this time period, when nonlinearity is fully in force, the nonlinear models predict the concentration in each compartment will increase at a much greater rate than does the linear model. In particular, the nonlinear model predicts the concentration in the softer trabecular bone will be over twice that in the harder cortical bone.

We now compare our results to those given in [2, Table 1]. There we see that the experimentally determined lead concentrations in subjects with no known occupational exposure to lead are,

(a) blood: $0.2 \pm 0.12 \mathrm{ppm}$

(b) cortical bone: $23.4 \pm 15.96 \mathrm{ppm}$

(c) trabecular bone: $8.85 \pm 5.81 \mathrm{ppm}$.

The nonlinear model then provides good results for the lead concentration in the blood and cortical bones. However, it does a poor job for the lead concentration in the trabecular bone. On the other hand, the linear model provides a reasonable result for the concentration in the trabecular bone, but it does not give a good prediction for the lead concentration in the cortical bone.

Consequently, we now refine our model by assuming the reaction rate for cortical bone to blood is nonlinear, but the reaction rate for trabecular bone to blood is linear. In other words, we set $P_{T, B}=0$. Still assuming $I=0.0255$, we get the results plotted in Figure 6. The top three panels give the relative amount of lead in each body compartment, whereas the bottom three panels give the lead concentration in each body compartment. Regarding the relative fractions, when comparing to the nonlinear solutions plotted in Figure 4 we see that the overall fraction of lead in the blood is essentially unchanged. On the other hand, the distribution in the bone is significantly different, with much more of it now being in the harder cortical bone. Regarding the concentrations plotted in the bottom three panels, when compared to the nonlinear solutions plotted in Figure 5 we see that the blood and cortical bone concentrations are essentially unchanged. On the other hand, the concentration of lead in the trabecular bone now follows the linear solution. In conclusion, the concentrations in each body compartment now give a reasonable prediction when compared to the data.

Remark 3.4. The data in [2, Table 1] shows that the bone in the skull (petrous temporal, and calvarium) contains an average of $53.8 \mathrm{ppm}$ of lead, which is over twice that contained in the cortical bone. Thus, it seems reasonable that we include this bone type in our ODE compartment model. Unfortunately, we do not at this time have exchange rate data for these bone types, and so we leave this task for further research. 

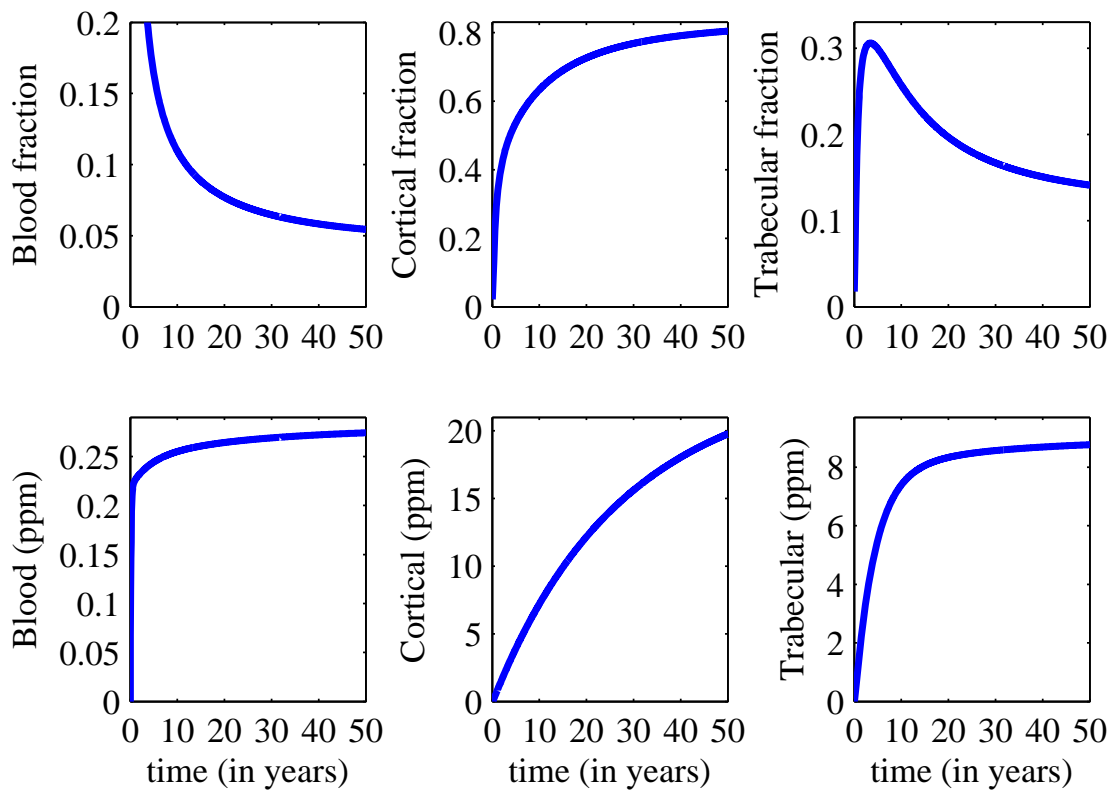

Figure 6: The data associated with the solution when the reaction rate from cortical bone to blood is nonlinear, but that from trabecular bone to blood is linear. The input rate is $I=0.0225$.

\section{THE PDE MODEL}

The final ODE model (nonlinear reaction rate for cortical bone, and linear reaction rate for trabecular bone) assumes that the concentration of lead is constant throughout a compartment. This seems to be a reasonable assumption for the blood; however, Marcus [16] suggests that in the bone there will be a slow diffusion process, with the diffusion constant being

$$
D_{0}=10^{-16} \frac{\mathrm{cm}^{2}}{\mathrm{sec}}=8.64 \times 10^{-4} \frac{\mu \mathrm{m}^{2}}{\text { day }} .
$$

Indeed, in [12, Figure 4] it is experimentally seen that for fossilized bone the rate of diffusion for lead is approximately $10^{-14} \mathrm{~cm}^{2} / \mathrm{sec}$. We now incorporate spatial effects into our model assuming the diffusion rate presented by Marcus. One perspective is that adding diffusion into the model is akin to breaking each bone type into two compartments - a surface compartment, and a volume compartment - without actually increasing the number of variables in the dynamical equations. We note that while we use the parameters from the beagle dog experiments in [16], we expect the results to be similar for humans given that observations for the distribution of lead in the body (such as $90 \%$ of the lead accumulating in the active bone matrix [10]) are also similar.

Following Marcus [16] we focus on a single canalicular territory for each bone type, which is modeled as a cylinder. The cylinders will be geometrically identical. Our coordinate system will be cylindrical, $(r, \theta, z)$. The cylinder will have an outer radius of $1 \mu \mathrm{m}$, and an inner radius of $a=0.088 \mu \mathrm{m}$. The blood interacts with the bone only in the inner portion of the cylinder, $0 \leq r \leq a$. The value of $a$ is chosen to be consistent with the ratio of inner radius to outer radius for a typical mouse canalicular territory. The cylinder will have length $L \mu \mathrm{m}$, i.e., $0 \leq z \leq L$, where $L$ is to be determined. We will assume that the amount of lead in the bone segment is independent of both $z$ and $\theta$; consequently, the dynamics happen only in the radial direction ([16] takes the same approach). While the blood interacts with the bone only in the inner cylinder, lead propagates in the outer portion of the canalicular territory, $a \leq r \leq 1$, through diffusion only. Marcus makes two additional assumptions in his model:

(a) lead ions transport instantaneously through the inner circle, and 


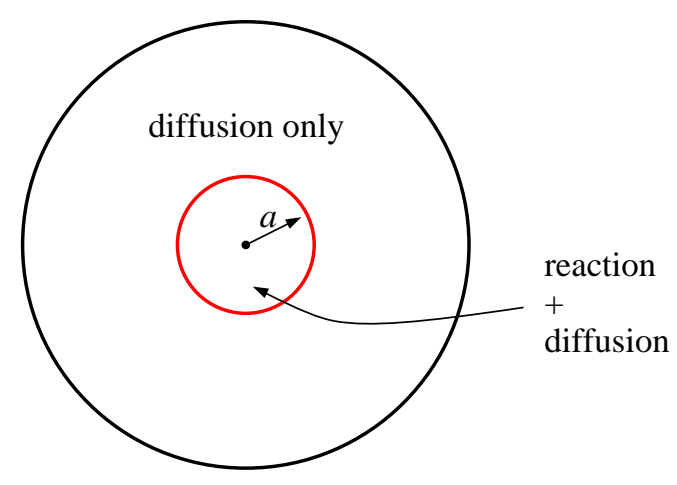

Figure 7: A cross-section of the canalicular territory. The outer radius has been normalized to have a value of one. The (red) inner radius is also renormalized to a value of $a=0.088$. The blood interacts with the bone only in the inner radius. The lead propagates in the outer region solely through a diffusion process.

(b) the inner radius is taken to be arbitrarily small, i.e., $a \rightarrow 0^{+}$.

Since the second assumption appears to be motivated solely by the solution approach, we do not make the same assumption. The goal here is to determine if adding a spatial component to the dynamics significantly affects the concentration of lead in each compartment.

Since the blood interacts with the bone only for $0 \leq r \leq a$, our dynamical equations will require the use of the Heaviside step function,

$$
H(r-a)= \begin{cases}0, & 0 \leq r<a \\ 1, & a \leq r .\end{cases}
$$

The system for cortical bone will be,

$$
\begin{aligned}
& \frac{\partial B}{\partial t}=D_{B} \Delta B-\left(k_{B, C}+k_{B, O}\right) B+k_{C, B} C+I \\
& \frac{\partial C}{\partial t}=D_{C} \Delta C+[1-H(r-a)]\left(k_{B, C} B-k_{C, B} C\right),
\end{aligned}
$$

and the system for trabecular bone will be,

$$
\begin{aligned}
& \frac{\partial B}{\partial t}=D_{B} \Delta B-\left(k_{B, T}+k_{B, O}\right) B+k_{T, B} T+I \\
& \frac{\partial T}{\partial t}=D_{T} \Delta T+[1-H(r-a)]\left(k_{B, T} B-k_{T, B} T\right),
\end{aligned}
$$

where $\Delta$ is the Laplacian (see Figure 7 for a cartoon of the domain). Here $k_{C, B}$ will be the nonlinear function derived in Section 2, whereas $k_{T, B}$ will be linear $\left(P_{T, B}=0\right)$. The function $B(r, t)$ is defined only for $0 \leq r \leq a$, but the spatial domain for each bone segment is $0 \leq r \leq 1$.

Consider the diffusion rates for the bones. Outside the canalicular territory, $a \leq r \leq 1$, it is known that cortical bone is denser than trabecular bone; for our purposes, we continue to choose $1.9 \mathrm{~g} / \mathrm{cm}^{3}$ for cortical bone, and $1.2 \mathrm{~g} / \mathrm{cm}^{3}$ for trabecular bone (the range is between $1.0-1.9 \mathrm{~g} / \mathrm{cm}^{3}$ ). Since the diffusion process in high density materials is generally slower than in low density materials, it is reasonable to assume that in this portion of the spatial domain, $D_{T}>D_{C}$. We will assume that the diffusion coefficient is proportional to the inverse of the density. Thus, we will assume that for $a \leq r \leq 1$,

$$
D_{T}=\frac{1.9}{1.2} D_{C} \sim 1.58 D_{C}
$$

In this region we will set $D_{C}=10^{-16} \mathrm{~cm}^{2} / \mathrm{sec}$. Inside the canalicular territory, where the reactions take place, we will assume that the diffusion rate is the same for both types of bone. Consequently, the diffusion 
rates will be,

$$
D_{C}=\left\{\begin{array}{ll}
D_{\text {in }}, & 0 \leq r<a \\
D_{0}, & a \leq r,
\end{array}, \quad D_{T}= \begin{cases}D_{\text {in }}, & 0 \leq r<a \\
1.58 D_{0}, & a \leq r,\end{cases}\right.
$$

where $D_{0}$ is given in (4.1). The diffusion rate for the inner radius, $D_{\text {in }}$, is a parameter, as the experimental diffusion rate for lead in the blood is unknown to us.

Since by assumption the ions transport (almost) instantaneously, the rate of diffusion for blood should be small compared to the reaction rate. We assume that the concentration of blood is independent of position, which means we set $D_{B}=0$. Regarding the bone fragments, we first note that the reaction rate is as slow as $\mathcal{O}\left(10^{-4}\right)$. Since we expect the reaction to dominate inside the canalicular territory, $0 \leq r \leq a$, we choose $D_{\text {in }} \leq 10^{-8} a^{2}$ (this is equivalent to a diffusion coefficient of $10^{-8}$ on the unit interval). For the sake of simulation we actually set $D_{\text {in }}=0$, so that there are only reaction dynamics in the inner cylinder. These choices are consistent with [16], in which it is assumed there is no diffusion in the inner cylinder. Numerical experiments indicate that the choice is not important, as long as it is sufficiently small. On the other hand, if the inner diffusion coefficient is of the same order as the reaction dynamics, then lead diffuses outside of the canalicular territory too quickly and does not allow for significant reaction dynamics.

Now we take care of the boundary conditions. Taking advantage of the circular geometry, we have for the Laplacian in radial coordinates,

$$
\Delta=\frac{\partial^{2}}{\partial r^{2}}+\frac{1}{r} \frac{\partial}{\partial r}
$$

Thus, for boundary conditions at the origin we need,

$$
\frac{\partial B}{\partial r}(0, t)=\frac{\partial C}{\partial r}(0, t)=\frac{\partial T}{\partial r}(0, t)=0 .
$$

The blood interacts with the bone only in the interior of the canalicular territory. Assuming no lead enters or leaves the blood at the interface,

$$
\frac{\partial B}{\partial r}(a, t)=0, \quad a=0.088 .
$$

As for the bone, we follow Marcus $[\mathbf{1 3}, \mathbf{1 6}]$ and assume that for some values of $c_{C}, c_{T}$,

$$
\frac{\partial C}{\partial r}(1, t)=-c_{C} C(1, t), \quad \frac{\partial T}{\partial r}(1, t)=-c_{T} T(1, t) .
$$

The underlying assumption here is that lead is removed from the surface of the canalicular territory at a rate proportional to the amount of lead on the surface.

Following Marcus $[\mathbf{1 3}, \mathbf{1 6}]$ we set $c_{C}=0.7$. We find numerically, however, that the exact value is not all that important. Since trabecular bone has a greater surface area than does cortical bone, it is more suitable for ion exchange; in particular, it implies that perhaps we should set $c_{T}>c_{C}$. Additional supporting evidence for such an assumption is provided in [18], where we are told that the rate loss for trabecular bone is roughly twice that for cortical bone. For the sake of simulation we set $c_{T}=1.2$.

Remark 4.1. The model assumes that the cortical bone and trabecular bone do not interact except via the intermediary of the blood in the reaction domain of the canalicular territory. However, it is reasonable to assume that there could be some interaction at the boundary, as the lead from one bone segment leaves to enter the other segment through the diffusion process. Incorporating this effect into the model is beyond our present scope, but it is certainly worth further study.

Finally, the assumed input rate into the entire body is $I=0.0225 \mathrm{mg} /$ day, which after assuming the body contains 5.4 liters of blood, and the lead is well mixed so that the concentration of lead in the blood is uniform, means that the incoming concentration rate is $4.167 \times 10^{-3} \mathrm{ppm} /$ day. Since the cylinder is of length $L \mu \mathrm{m}$, this means that the incoming rate of lead in the cylinder is,

$$
I=\left(\pi a^{2} L \mu \mathrm{m}^{3}\right)\left(4.167 \times 10^{-3} \frac{\mathrm{ag}}{\mu \mathrm{m}^{3}}\right)=\left(1.014 \times 10^{-5}\right) L \mathrm{ag}, \quad 1 \mathrm{ag}=10^{-15} \mathrm{mg} .
$$

We have no data to inform us as to what $L$ should be; however, an examination of visual images (found via a Google search) suggests it is not unreasonable to choose $L=100 \mu \mathrm{m}$. Thus, with the cylinder of internal 
radius $a \mu$ and length $100 \mu \mathrm{m}$ we have the input rate into the cylinder is,

$$
I=1.014 \times 10^{-3} \frac{\mathrm{ag}}{\mathrm{day}} .
$$

Going back to the transfer rates illustrated in Figure 2, we see that for this value of input rate the reaction dynamics will be linear. Consequently, the expectation is that at best the concentration levels in the cylinder will roughly match the (red) dashed curves presented in Figure 5. In particular, if the PDE micro model and the ODE compartment model are to roughly agree we expect that after many years the lead concentration in the blood will be approximately $0.25 \mathrm{ppm}$, and the lead concentration in the cortical bone will be approximately $10 \mathrm{ppm}$.

\subsection{Simulation results}

While we cannot solve the nonlinear systems (4.2) and (4.3) analytically, we can solve them numerically. We approximate the spatial derivatives through a standard second-order finite-difference scheme. Since the diffusion coefficients for the bone segments are spatially dependent, the step size is not uniform. In particular, the step for $0 \leq r<a$ will be much smaller than that for $a \leq r \leq 1$, as the diffusion coefficient over the inner canalicular territory is assumed to be much smaller than that outside the canalicular territory. Once the PDEs are transformed into a large number of nonlinear ODEs, we use MATLAB's ode15s to solve the system. We made this choice because of the potential stiffness problems associated with having a piecewise constant diffusion coefficient.
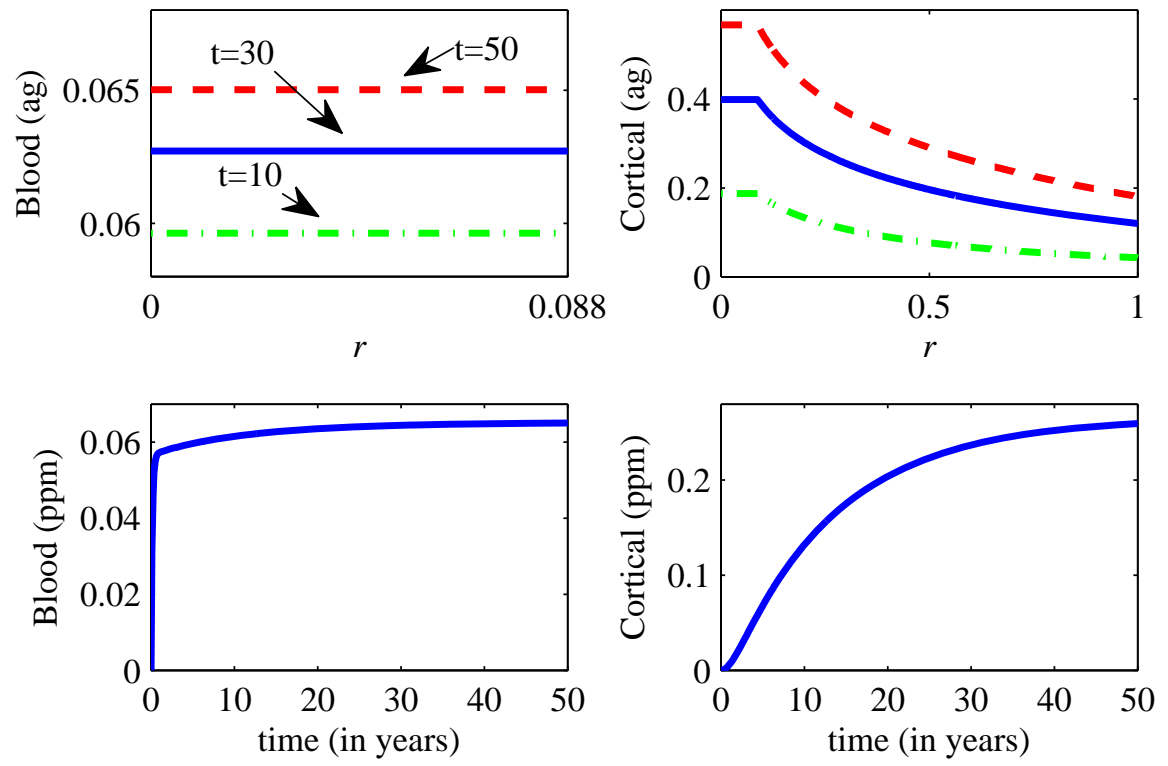

Figure 8: The top two panels provide a sequence of time plots for the blood-cortical model of (4.2) with $I=1.014 \times 10^{-3} \mathrm{ag} /$ day. The dot-dashed (green) curve corresponds to the solution after 10 years, the solid (blue) curve corresponds to the solution after 30 years, and the dashed (red) curve corresponds to the solution after 50 years. The bottom two panels provide the lead concentrations in ppm for blood and cortical bone.

The results of the simulation for the blood-cortical model of (4.2) are shown in Figure 8. The top two panels provide time snapshots at $t=10,30,50$ years, while the bottom two panels give the lead concentration ppm. Using the assumption that the amount of lead is unchanged along the cylinder, the concentration for 
lead in the cortical bone was calculated via,

$$
C_{\text {conc }}(t)=\frac{2 \pi L \int_{0}^{1} r C(r, t) \mathrm{d} r}{\pi L} \frac{\mathrm{ag}}{\mu \mathrm{m}^{3}}=\left(2 \int_{0}^{1} r C(r, t) \mathrm{d} r\right) \mathrm{ppm},
$$

and the concentration for lead in the blood is,

$$
B_{\text {conc }}(t)=\frac{2 \pi L \int_{0}^{a} r B(r, t) \mathrm{d} r}{\pi a^{2} L} \frac{\mathrm{ag}}{\mu \mathrm{m}^{3}}=\left(\frac{2}{a^{2}} \int_{0}^{a} r B(r, t) \mathrm{d} r\right) \mathrm{ppm} .
$$

In both cases the concentration is independent of the length of the cylinder; consequently, the length only affects the input rate of lead in this model. Note that the numerical value of the concentration for blood and cortical bone correspond to the spatial averages of $B(r, t)$ and $C(r, t)$, respectively.

First consider the top two panels. As expected, we see that the level of lead in the blood is constant as a function of distance from the origin. Regarding the lead level in the cortical bone, we see that the level is constant for $0 \leq r \leq a$, where there are reaction dynamics with the blood, and then there is a transition at the boundary to a smaller level which decreases as a function of distance from the center of the cylinder. The decrease in lead levels is due to the diffusion process. Note that the amount of lead at the boundary of the cylinder, $r=1$, increases as a function of time, but is always a minimum. This is due to the boundary condition, which assumes that lead is drawn out of the bone.

\subsection{Comparison to ODE model}

We now compare the bottom two panels of Figure 8 with the bottom left and center panel of Figure 6 . We first note that the curves have the same qualitative features; in particular, the concentration in the blood quickly reaches a pseudo-steady state value, and thereafter increases very slowly, while the concentration in the cortical bone increases steadily throughout. At the end of 50 years the concentration in the bone is roughly four times greater than that in the blood. On the other hand, the curves do not at all coincide quantitatively. The concentration in the cortical bone is approximately only $1 / 50$ of what the linear ODE model (and data) predicts, and the concentration in the blood is approximately only $1 / 4$ of what the linear ODE model (and data) predicts.

Unlike [16], we do not find good agreement between the ODE model and the PDE model. We think there are (at least) three possible reasons why the models do not agree:

(a) From a mathematical perspective the input rate for the ODE model is over 22 times larger than that for the PDE model. Of course, as seen in (4.5) the input rate for the PDE model can be adjusted simply by changing the length of the cylinder. However, to get a comparable input rate would require a length of cylinder that, to us, appears to be unreasonable. Indeed, even if we choose the length so that the input rates are the same, then we find that the blood concentration after 50 years is $1.4 \mathrm{ppm}$, which is about 6 times that predicted by the ODE model, whereas the cortical blood concentration after 50 years is $9.5 \mathrm{ppm}$, which is about one-half of what the ODE model predicts.

(b) The reaction rates are derived using macro data; in particular, treating the body as being composed of compartments, and then looking at the rate of transfer between the various compartments. On the other hand, the cortical bone PDE model is a micro model, and it may be the case that the transfer rates used in the reaction dynamics are not only a function of amount of lead in the entire compartment, but also a function of the size of the compartment. In other words, the transfer rates for the macro compartment model may not be the same as those for the micro PDE model.

(c) We assume the rate of diffusion is independent of the concentration of lead in the bone. This may be incorrect; indeed, it may be better to actually assume the diffusion rate decreases as a function of lead amount. This assumption would allow for more of the lead to remain in the inner cylinder, which would increase the amount of reaction taking place. 
Regarding point (a), it is not clear from [16] how the input rate was chosen for his PDE model. Regarding point (b), it interestingly appears to be the case that for [16] this was not an issue.

Since the blood-cortical bone PDE model (4.2) does not match the experimental data well, we do not present the results of the simulation for the blood-trabecular bone PDE model (4.3). We find, however, that it also greatly underestimates the concentrations in each compartment.

\section{COnClusions And Further Work}

We explored a three-compartment ODE model describing how lead is transported between blood and two different types of bone in the body. The ODE model is a functionally standard compartment model $([4,20])$, but we include nonlinearity in our analysis to account for the change in transfer rates as the amount of lead in the body increases [4]. We demonstrate that the nonlinear interactions affect:

(a) the total amount of lead in the body, yielding a greater amount of lead for reasonable input rates

(b) the relative fraction of lead in each compartment; in particular, a greater percentage of the lead will be in the bone

(c) the concentration of lead in each compartment; in particular, there is better agreement with experimental results.

Finally, we showed that there is reasonable agreement with the experimental data if we assume that the cortical bone to blood interaction is nonlinear, but the trabecular bone to blood interaction is linear.

We then considered spatial effects by adding diffusion. Following Marcus [16], the spatial domain for each bone segment was assumed to be a cylinder. Assuming that the amount of lead was not a function of distance along the cylinder, we (re-)derived a PDE for blood-cortical bone interactions, and a PDE for bloodtrabecular bone interactions. We assume that there is only the nonlinear ODE reaction in the inner radius, and diffusion only in the outer annulus. The diffusion rate in the outer radius is known experimentally. We concluded by showing that the nonlinear ODE model and PDE model were not in agreement, either qualitatively or quantitatively.

Natural extensions of this work include considering a realistic time-varying lead ingestion rate instead of a constant rate, as well as adding tissue compartments (neural, digestive) to the model to explore if they have any significant effect on the body lead concentration, and the manner in which lead storage in the bone affects the lead levels in these compartments. Regarding the PDE model, perhaps it can be improved to yield more realistic results by allowing for many cylinders where not only there are reaction dynamics within each cylinder, but there is also interaction between the cylinders through the boundary. In particular, the model should allow for lead from one cylinder to migrate to another cylinder via the diffusion process. This may be an important effect in allowing the amount of lead in the bone to increase; indeed, we find that by setting $c_{C}=0.07$,

(a) which is $1 / 10$ of the original value, and

(b) allows for much less lead to leave the bone fragment via diffusion,

then the concentration of lead in the bone fragment almost doubles, whereas the concentration of lead in the blood is essentially unchanged. Finally, our ODE and PDE models have the potential to be incorporated with physiologically based studies of lead kinetics that take into account differences between genders or account for bone formation in children [17].

Acknowledgments. This paper is based upon work supported by The Institute of Computational and Experimental Research in Mathematics at Brown University. Thank you to our advisor Professor Todd Kapitula for his guidance, and to our Graduate Teaching Assistant Veronica Ciocanel. We would also like to thank Professor Björn Sandstede for his help and support. 


\section{REFERENCES}

[1] Fernando Barbosa, Irene Ramires, Maria Heloísa C Rodrigues, Tatiana D Saint-Pierre, Adilson J Curtius, Marilia R Buzalaf, Raquel F Gerlach, and José E Tanus-Santos. Contrasting effects of age on the plasma/whole blood lead ratio in men and women with a history of lead exposure. Environmental Research, 102(1):90-95, 2006.

[2] P. Barry. A comparison of concentrations of lead in human tissues. British Journal of Industrial Medicine, 32(2):119-139, 1975.

[3] E Batschelet, L Brand, and A Steiner. On the kinetics of lead in the human body. Journal of Mathematical Biology, 8(1):15-23, 1979.

[4] Sepideh Behinaein, David R Chettle, Lesley M Egden, Fiona E McNeill, Geoff Norman, Norbert Richard, and Susan Stever. The estimation of the rates of lead exchange between body compartments of smelter employees. Environmental Science: Processes 6 Impacts, 16(7):1705-1715, 2014.

[5] JL Bert, LJ Van Dusen, and JR Grace. A generalized model for the prediction of lead body burdens. Environmental Research, 48(1):117-127, 1989.

[6] Adele L Boskey and Rhima Coleman. Aging and bone. Journal of Dental Research, 89(12):1333-1348, 2010.

[7] José AA Brito, Fiona E McNeill, David R Chettle, Colin E Webber, and Claude Vaillancourt. Study of the relationships between bone lead levels and its variation with time and the cumulative blood lead index, in a repeated bone lead survey. Journal of Environmental Monitoring, 2(3):271-276, 2000.

[8] B. Clarke. Normal bone anatomy and physiology. Clin. J. Am. Soc. Nephrol., 3:S131-S139, 2008.

[9] Gagan Flora, Deepesh Gupta, and Archana Tiwari. Toxicity of lead: a review with recent updates. Interdiscip Toxicol, 5(2):47-58, 2012.

[10] Ramesh C. Gupta. Veterinary toxicology: basic and clinical principles. Academic Press, 2012.

[11] Todd Kapitula. Ordinary Differential Equations and Linear Algebra: A Systems Approach, volume 145. SIAM, 2015 .

[12] Matthew J Kohn and Randolph J Moses. Trace element diffusivities in bone rule out simple diffusive uptake during fossilization but explain in vivo uptake and release. Proceedings of the National Academy of Sciences, 110(2):419-424, 2013.

[13] Allan H Marcus. Compartmental models with spatial diffusion: estimation for bone-seeking tracers. Mathematical Biosciences, 64(2):233-248, 1983.

[14] Allan H Marcus. Multicompartment kinetic model for lead: III. Lead in blood plasma and erythrocytes. Environmental Research, 36(2):473-489, 1985.

[15] Allan H Marcus. Multicompartment kinetic model for lead: II. Linear kinetics and variable absorption in humans without excessive lead exposures. Environmental Research, 36(2):459-472, 1985.

[16] Allan H Marcus. Multicompartment kinetic models for lead: I. Bone diffusion models for long-term retention. Environmental Research, 36(2):441-458, 1985.

[17] E. O'Flaherty. A physiologically based kinetic model for lead in children and adults. Environmental Health Perspectives, 106 (Suppl 6):1495, 1998.

[18] E. O'Flaherty. Modeling normal aging bone loss, with consideration of bone loss in osteoporosis. Toxicological Sciences, 55:171-188, 2000.

[19] M. Rabinowitz. Historical perspective on lead biokinetic models. Environmental health perspectives, 106(Suppl 6):1461, 1998.

[20] Michael B Rabinowitz, George W Wetherill, and Joel D Kopple. Kinetic analysis of lead metabolism in healthy humans. Journal of Clinical Investigation, 58(2):260, 1976. 
[21] Providence Water. Important information about lead in your drinking water. Annual Report, 552 Academy Avenue, RI 02908, 2015.

[22] U.S. EPA. Review of adult lead models: evaluation of models for assessing human health risks associated with lead exposures at non-residential areas of Superfund and other hazardous waste sites. U.S. Environmental Protection Agency Technical Review Workgroup for Lead. EPA/OSWER \#9285.7-46, 2001.

[23] Lorentz E Wittmers Jr, Joann Wallgren, Agnes Alich, Arthur C Aufderheide, and George Rapp Jr. Lead in bone. iv. distribution of lead in the human skeleton. Archives of Environmental Health: An International Journal, 43(6):381-391, 1988. 